\title{
Humans Trade Off Viewing Time and Movement Duration to Improve Visuomotor Accuracy in a Fast Reaching Task
}

\author{
Peter W. Battaglia ${ }^{1}$ and Paul R. Schrater ${ }^{1,2}$ \\ Departments of ${ }^{1}$ Psychology and ${ }^{2}$ Computer Science, University of Minnesota, Twin Cities, Minneapolis, Minnesota 55455
}

Previous research has shown that the brain uses statistical knowledge of both sensory and motor accuracy to optimize behavioral performance. Here, we present the results of a novel experiment in which participants could control both of these quantities at once. Specifically, maximum performance demanded the simultaneous choices of viewing and movement durations, which directly impacted visual and motor accuracy. Participants reached to a target indicated imprecisely by a two-dimensional distribution of dots within a 1200 ms time limit. By choosing when to reach, participants selected the quality of visual information regarding target location as well as the remaining time available to execute the reach. New dots, and consequently more visual information, appeared until the reach was initiated; after reach initiation, no new dots appeared. However, speed accuracy trade-offs in motor control make early reaches (much remaining time) precise and late reaches (little remaining time) imprecise. Based on each participant's visual- and motor-only targethitting performances, we computed an "ideal reacher" that selects reach initiation times that minimize predicted reach endpoint deviations from the true target location. The participant's timing choices were qualitatively consistent with ideal predictions: choices varied with stimulus changes (but less than the predicted magnitude) and resulted in near-optimal performance despite the absence of direct feedback defining ideal performance. Our results suggest visual estimates, and their respective accuracies are passed to motor planning systems, which in turn predict the precision of potential reaches and control viewing and movement timing to favorably trade off visual and motor accuracy.

Key words: vision; motor control; decision; optimality; visuomotor variability; timing

\section{Introduction}

Sensorimotor decisions involve three distinct components: world states, potential actions, and the rewards associated with different combinations of the first and second components. Inferring the state of the world is an implicitly uncertain process because of incomplete and noisy sensory data, and the outcome of intended actions is inherently variable for reasons that include neural firing variability and imprecise muscle responses to motor commands. Recent studies reveal the brain contains a rich representation of these components of sensorimotor decisions, including perceptual uncertainty, expected motor output variability, and monetary rewards (Trommershäuser et al., 2003a,b; Wu et al., 2006). Because states of the world are often dynamic and actions unfold in time, decisions must also take time into account. Trommershäuser et al. (2005, 2006a,b) showed that such near-optimal target selection may occur under tight time constraints and even in cases when the target or reward information vary during the reach. Our study asks: does the brain represent visual and motor variability as functions of time? Moreover, when task perfor-

Received Dec. 20, 2006; revised May 7, 2007; accepted May 8, 2007.

This work was supported by National Institutes of Health Grant R01-EY015261, Office of Naval Research Grant N00014-05-1-0124, and the National Science Foundation Graduate Research Fellowship program. We thank Dr Daniel Kersten for valuable advice regarding the experimental design and this manuscript. We also thank our reviewers for insightful comments and suggestions for revisions.

Correspondence should be addressed to Peter W. Battaglia, Department of Psychology, University of Minnesota, Twin Cities, Elliott Hall, 75 East River Road, Minneapolis, MN 55455. E-mail: batt0086@umn.edu.

DOI:10.1523/JNEUROSCI.1309-07.2007

Copyright $\odot 2007$ Society for Neuroscience $\quad$ 0270-6474/07/276984-11\$15.00/0 mance is degraded by both visual and motor errors, can the brain manipulate the sources of these errors to maximize task performance?

Many visually guided motor behaviors have time constraints to completion that induce a tradeoff between time allotted to gather sufficient visual information for action planning and time allotted for action execution. Often there is exactly one action to be performed within a finite time; competition for the limited time allowed by the task requires the simultaneous choice of viewing and movement durations. Longer viewing durations improve the quality of visual information, whereas longer movement durations decrease motor errors, and thus the choices of these durations directly impact performance.

For example, consider driving on a mountain road during a snowstorm. As visual information accrues suggesting a possible obstacle ahead, like a boulder or car, a plan must rapidly be formed to continue on course, slam the brakes, or perform a risky swerve. Viewing and action time intervals are controllable, and their durations directly affect task performance. Waiting lets you base your decision on greater sensory information but leaves less time to execute the action. Reacting immediately may allow sufficient time to execute the action but affords little information to decide which action is best. Therefore, time-constrained visuomotor tasks require balancing visual and motor timing to minimize visual and motor errors. The goal of this study is to psychophysically test how well people can achieve the optimal performance tradeoff in an experimentally controlled visuomotor task. 
We asked participants to reach to a target within a fixed time limit. Our experiment was designed to allow participants to trade visual accuracy for motor accuracy by choosing when to initiate their reaches. We evaluated how participants balanced this tradeoff to infer whether they knew, and were able to control, their visual and motor variability.

\section{Materials and Methods \\ Apparatus}

Participants performed trials in a virtual workbench consisting of the graphical display of a scene rendered under accurate perspective projection and a haptic interface (PHANToM force-feedback device; Sensable Technologies, Woburn, MA) that simulated the feel of objects in the scene. The graphics were displayed on a 21 -inch CRT monitor (pixel resolution, $1600 \times 1200 ; 85 \mathrm{~Hz}$ ). The image of the monitor was reflected off a full-silvered mirror so that the graphics appeared coaligned with the haptic workspace. The graphics and haptics were calibrated to always be consistent with the three-dimensional virtual scene. The virtual scene consisted of a frontoparallel task surface with contour information to enrich perspective cues, a start button, a countdown sand-timer, and the target stimulus. Participants wore eye patches over their left eyes to remove potential stereodisparity cue conflict. Participants placed their fingertips in a thimble attached to the haptic interface, which tracked the fingertip position. Visual feedback of fingertip position was continuously provided as a 1.3 -mm-diameter sphere.

\section{Target}

On each trial, our participants' task was to place his or her fingertip on a 2.5-mm-diameter "start button" and reach to a $2.75 \mathrm{~mm}$ arc-length target (Fig. 1). The target itself was invisible but always lay collinear with a visible, gray "guide arc," which was an arc-length at a radius $8 \mathrm{~cm}$ from the start button (arc interval between 176 and $236^{\circ}$ counterclockwise from the positive $x$-axis). Also, the position of the target was indicated by a dynamic visual stimulus that appeared at the start of each trial. The stimulus was composed of very small dots scattered around the target position by sampling their positions from a two-dimensional (2D) normal distribution with a mean at the target position and with a SD (termed dot scatter level) that was varied across conditions. Three dot scatter levels were used: 4, 7, and $11 \mathrm{~mm}$ (low, medium, and high, respectively).

At the trial start, there were always five dots visible. As the trial time elapsed, the number of visible dots increased quadratically until a reach was initiated. The number of dots at any particular elapsed trial time was given by the following:

$$
N(t)=\text { floor }\left[\left(t^{\star}\left(\frac{5.47}{1200}\right)+2.45\right)^{2}-1\right],
$$

where floor $(x)$ rounds $x$ down to the nearest integer, $t$ is elapsed trial time, and $N(t)$ is the number of dots as a function of $t$.

An ideal observer simply computes the sample mean of the dot positions as the optimal target location estimate. Therefore, the ideal observer's SD from the true target location decreases linearly with the square root of $N(t)$. We chose the quadratic relationship between $N(t)$ and $t$ so that the ideal observer's SD from the true target location decreased linearly with $t$.

\section{Procedure: Experiment 1}

Three conditions were run: combined test condition (CC), visual baseline (VB), and motor baseline (MB). The participant's task varied slightly between conditions. Before each block, participants performed a series of practice trials to familiarize them with the task.

Practice sessions. Before every block in every condition, participants were given $\sim 50$ practice trials that were identical to the trials of the subsequent block but were not recorded or further analyzed. The purpose of these practice trials was to allow the participant to warm up and familiarize him or herself with the particular trial procedure of the subsequent block.

Combined test condition. In each trial, the participant held the fingertip on the start button for $500 \mathrm{~ms}$ (Fig. 1) to signal he or she was ready to

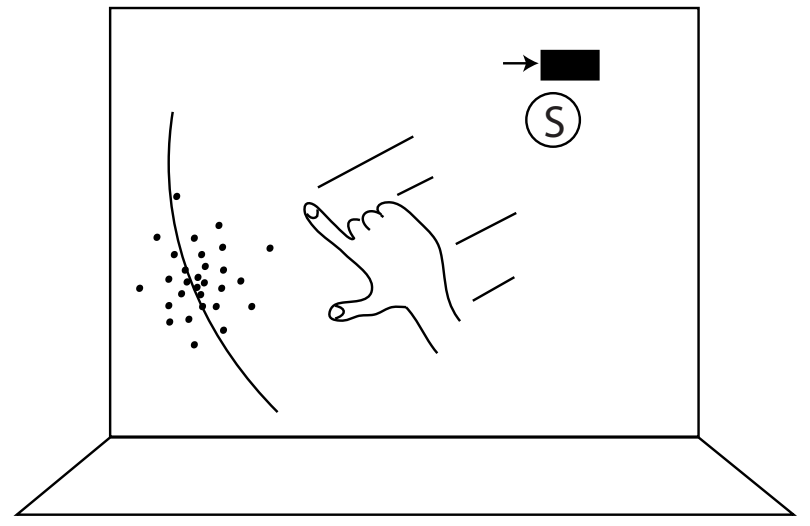

Figure 1. Experimental diagram. In each trial, the participant placed his or her fingertip on the start button (circle labeled " $S$ ") to signal he or she was ready to begin the trial. The trial then started, and the countdown sand-timer (rectangle above start button) appeared and began to decrease in width (indicated by an arrow). Also, the invisible target was randomly positioned on the guide arc to the left. The scattered dots began to appear, which provided an uncertain visual cue to the location of the target.

begin. When the computer detected the ready state, the trial started. At the trial start, the invisible target was placed at a random location on the guide arc. Simultaneously, a rectangular sand-timer appeared above the start button and began counting down the fixed trial time (1200 ms). The width of the sand-timer was always proportional to the remaining trial time, thus providing a visual cue to the remaining trial time.

To complete a reach before timing out, the participant had to move the fingertip from the start button to the guide arc within $1200 \mathrm{~ms}$ of the trial start. The time at which the fingertip first contacted the guide arc was called the trial end. If the participant did not complete his or her reach within the time limit, the trial was considered a "timeout," and repeated later with a novel stimulus and target location. To make a successful reach, the participant had to be in contact with the target at the trial end. No matter what, the trial always ended once the fingertip first contacted the guide arc, meaning the participant could not cross the guide arc and then return to cross it again at another location. The computer recorded the position at which the fingertip first crossed the guide arc; this was considered to be the participant's indication of the target position for that trial. The distance, measured along the guide arc in millimeters, between the center of the target and the position at which the finger first crossed the guide arc is termed the reach endpoint offset and signifies the spatial inaccuracy of a reach. Because the target was a $2.75 \mathrm{~mm}$ arclength, any reach endpoint offset $\leq 1.375 \mathrm{~mm}$ was considered successful, whereas any offset $>1.375 \mathrm{~mm}$ was considered unsuccessful.

The computer also recorded the duration of two time intervals during each trial, viewing time $\left(t_{\mathrm{v}}\right)$ and movement time $\left(t_{\mathrm{m}}\right) \cdot t_{\mathrm{v}}$ was the interval between trial start and the reach initiation time, when the fingertip left the start button to move toward the target. $t_{\mathrm{m}}$ was the interval between the reach initiation time and the trial end, when the fingertip first contacted the guide arc. The computer recorded the $t_{\mathrm{v}}$ and $t_{\mathrm{m}}$ durations with a temporal resolution of $11.8 \mathrm{~ms}$.

Participants ran six blocks of 150 trials each. There were three dot scatter level subconditions (low, medium, and high), with two blocks in each subcondition. Each block took $\sim 20$ min to complete and was divided into 30 trial sub-blocks. Between sub-blocks, participants were allowed to rest for a few minutes before continuing. Also, the participant was informed of the cumulative number of successful trials, in which the fingertip had contacted the target, for that block. This was the only form of feedback about task performance provided to the participants during the CC condition of Experiment 1.

Visual baseline condition. The VB condition was designed to quantify the relationship between the number of visible dots and visual variability for each participant, without any effect of motor variability. The visual stimulus presentation that indicated the target location was similar to the CC condition, but instead new dots continued to appear after the reach was initiated until a predetermined time that was varied between sub- 
blocks. Therefore, participants could freely move their fingertips without affecting the appearance of the visual stimulus. Also, to indicate the target location, the participant positioned the fingertip on his or her estimate of the target location and depressed the left mouse button. Participants in the $\mathrm{VB}$ condition were given $5000 \mathrm{~ms}$ to complete the task (instead of the $1200 \mathrm{~ms}$ in the CC condition), which was more than adequate time to precisely position their fingertip. Participants rarely used the full 5000 ms; typically, they made target location selections within 2000-3000 ms. Effectively, these alterations eliminated target localization imprecision because of motor variability so that we could isolate the effect of the number of dots on visual variability. The computer recorded $t_{\mathrm{v}}$ as the predetermined duration of the interval over which new dots appeared as well as reach endpoint offsets, which were analyzed to determine their relationship.

Participants ran three blocks of 150 trials each. There were three dot scatter level subconditions (low, medium, and high), with one block in each subcondition. Each block took $\sim 20$ min to complete and was divided into 30 trial sub-blocks. The time intervals over which new dots appeared varied across sub-blocks. From first to fifth sub-blocks, the time intervals over which new dots appeared were $0,212,425,637$, or 850 ms or a total of $5,11,19,28$, or 39 dots, respectively. Between sub-blocks, participants were allowed to rest for a few minutes before continuing. Also, the participant was informed of the cumulative number of trials in which he or she had completed a successful reach for that block. This was the only form of feedback about task performance provided to the participants during the VB condition of Experiment 1.

Motor baseline condition. The MB condition was designed to quantify the relationship between movement duration and motor variability for each participant, without any effect of visual variability. The MB condition was similar to the CC condition, with several differences. Instead of scattered dots, the visual stimulus that indicated the target location was now a small $2.75 \mathrm{~mm}$ white arc-length that perfectly specified the target location. Also, the trial time limit was not constant across the MB condition, it was varied between sub-blocks, and was always $\leq 1200 \mathrm{~ms}$. The initial width of the countdown sand-timer was shortened to match the trial time limit of the sub-block. Once the trial started, the sand-timer shortened at the same rate as in all other conditions so that the width was proportional to the remaining trial time. Also, the trial start was determined by the reach initiation time, that is, trial time began to elapse once the reach was initiated. The participant was instructed to use as much time as possible to complete his or her reach. Effectively, these alterations removed the effects of visual target localization variability to isolate the relationship between movement duration and motor variability. The computer recorded $t_{\mathrm{m}}$ and reach endpoint offsets, which were analyzed to determine their relationship. Note that in the analysis, we related reach precision to the measured $t_{\mathrm{m}}$, not the cued movement time.

Participants ran two blocks of 150 trials each. Each block took $\sim 20$ min to complete and was divided into 30 trial sub-blocks. The cued movement time varied across the five sub-blocks. From the first to fifth sub-blocks, the cued movement times were $1200,988,775,563$, and 350 $\mathrm{ms}$. Between sub-blocks, participants were allowed to rest for a few minutes before continuing. Also, the participant was informed of the cumulative number of trials in which he or she had completed a successful reach for that block. This was the only form of feedback about task performance provided to the participants during the $\mathrm{MB}$ condition of Experiment 1.

\section{Procedure: Experiment 2}

We conducted Experiment 2 to assess the effect of performance feedback on reaching behavior. Experiment 2 was similar to Experiment 1 with several key differences. First, participants received performance feedback after every trial, in addition to the sub-block success summaries. Performance feedback consisted of the haptic sensation of a bump when the target had been contacted, as well as visual presentation of an illuminated arc-length at the location of the target that was green if the target had been successfully contacted and red if the target had been missed. Second, we did not include the medium dot scatter level condition, thus only the 4 and $11 \mathrm{~mm}$ dot scatter levels were used. Third, two Experiment 1 participants were unavailable, so only five of the original seven participants participated in Experiment 2.

In Experiment 2, we repeated the VB and MB conditions identically to Experiment 1 (no performance feedback) for each participant and compared performance with Experiment 1.

Participants and compensation. Seven (four females, three males) naive, right-handed University of Minnesota students with normal or corrected-to-normal vision participated in this experiment. Participants gave informed consent in accordance with University of Minnesota Institutional Review Board standards, were compensated $\$ 8.00 / \mathrm{h}$ of participation and received bonus money depending on performance.

Participants could earn bonus money in all conditions. Whenever the participant's reach contacted the target, the trial was considered a success. For each successful trial, the participant earned $\$ 0.02$ bonus. This bonus money was awarded in addition to the $\$ 8.00 / \mathrm{h}$ compensation for participation time.

\section{Model}

Visual and motor accuracy tradeoff. In each CC trial, once the reach was initiated, no new dots appeared, meaning the visual stimulus no longer improved. Therefore, the reach initiation time effectively divided the trial into two distinct intervals: viewing time and movement time. Because visual localization accuracy improved with increased viewing time, $t_{\mathrm{v}}$, and reach precision improved with increased movement time, $t_{\mathrm{m}}$, the reach initiation time implicitly imposed a tradeoff between visual and motor accuracy. Ideal CC condition performance required the participant to select $t_{\mathrm{v}}$ and $t_{\mathrm{m}}$ to jointly maximize visual and motor accuracy.

To make predictions for participants' maximal CC performances, we computed an "ideal reacher" by combining participants' individual predicted visual and motor variability, measured in their VB and MB conditions, respectively. Figure 2 depicts ideal predicted CC performance. Each box in Figure 2 shows a different dot scatter level condition. For illustrative purposes, Figure 2 constrains $t_{\mathrm{m}}$ to be equal to $\left(1200-t_{\mathrm{v}}\right)$ so that $t_{\mathrm{v}}$ and $t_{\mathrm{m}}$ can be represented on a single axis. Notice how the tradeoff between visual and motor accuracy produces predicted CC performance curves that vary with viewing time, $t_{\mathrm{v}}$. The viewing time that minimizes predicted CC performance function (Fig. 2, arrows with numbers) increases as dot scatter level increases.

Ideal reacher derivation. CC condition reaches were modeled as follows. The process of planning and executing a reach included two components. First, the participant visually estimated the location of the target. Let $X$ represent the true target location and $\hat{X}$ represent the visually estimated target location. We assumed there was visual variability that contributed to additive errors, $\varepsilon_{v}$, between $X$ and $\hat{X}$ such that $\hat{X}=X+\varepsilon_{v}$. Second, the participant directed a reach toward the estimated location. Let $Z$ represent the reach endpoint. We assumed there was motor variability that contributed to additive errors, $\varepsilon_{\mathrm{M}}$, between $\hat{X}$ and $Z$ such that $Z=\hat{X}+\varepsilon_{\mathrm{M}}$. Together,

$$
Z=X+\varepsilon_{\mathrm{V}}+\varepsilon_{\mathrm{M}}
$$

resulting in a total error, $\varepsilon_{\mathrm{C}}=\varepsilon_{\mathrm{V}}+\varepsilon_{\mathrm{M}}$, that constitutes the reach endpoint offset between $Z$ and $X$.

Visual errors, $\varepsilon_{\mathrm{V}}$, varied with $t_{\mathrm{V}}$ and $\sigma_{\mathrm{d}}$, had mean equal to 0 , and variance, $\sigma_{\mathrm{v}}{ }^{2}$, such that

$$
\sigma_{\mathrm{V}}\left(t_{\mathrm{V}}, \sigma_{\mathrm{d}}\right)^{2}=\left(\rho_{\mathrm{e}}^{2}+1\right) \frac{\sigma_{\mathrm{d}}^{2}}{N\left(t_{\mathrm{V}}\right)}+2 \rho_{\mathrm{e}} \omega_{\mathrm{e}} \frac{\sigma_{\mathrm{d}}}{\sqrt{N\left(t_{\mathrm{v}}\right)}}+\omega_{e}^{2},
$$

where $\rho_{\mathrm{e}}$ and $\omega_{\mathrm{e}}$ are free parameters that were fit by maximum likelihood estimation (MLE) to the VB data. The derivation of Equation 3 and the fitting procedure are described in the Appendix. Note that for the rest of this study, we will suppress explicit dependency expressions after their initial presentation; for instance, $\sigma_{\mathrm{v}}\left(t_{\mathrm{v}}, \sigma_{\mathrm{d}}\right)^{2}$ will simply be written $\sigma_{\mathrm{v}}^{2}$.

Motor errors, $\varepsilon_{\mathrm{M}}$, varied with $t_{\mathrm{M}}$, had mean equal to 0 , and variance, $\sigma_{\mathrm{M}}^{2}$, such that

$$
\sigma_{\mathrm{M}}\left(t_{\mathrm{M}}\right)=D \cdot \exp \left(\alpha-\beta t_{\mathrm{M}}\right)+\gamma
$$

where $\alpha, \beta$, and $\gamma$ are free parameters that were fit by MLE to the MB data, and $D$ was the distance between the start button and the guide $\operatorname{arc}(8$ 


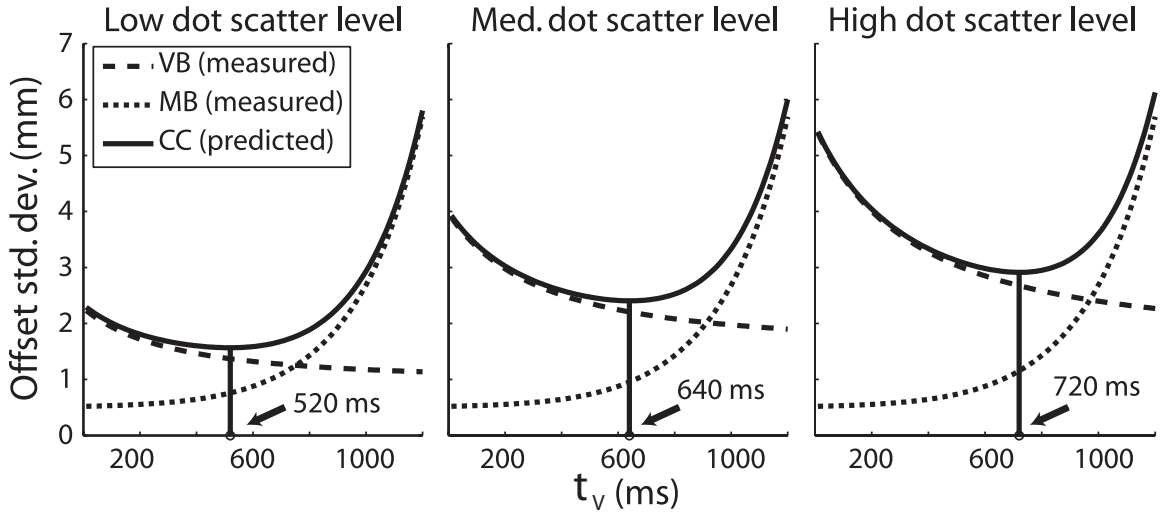

Figure 2. Visual, motor, and predicted CC performance versus elapsed time (participant 4). Visual accuracy, motor accuracy, and predicted $C(\mathrm{Condition}$ reach endpoint offsets (dotted, dashed, and solid lines, respectively) are plotted as functions of reach initiation time ( $x$-axis) for participant 4 . For the VB and $C\left(C\right.$ curves, reach initiation time is equivalent to $t_{\mathrm{v}}$. For the $\mathrm{MB}$ curve, reach initiation time is equivalent to $\left(1200-t_{\mathrm{M}}\right)$ and assumes that the participant used all of the remaining trial time after leaving the start button as $t_{M}$, so that he or she finishes the reach at a trial time of $1200 \mathrm{~ms}$. The $y$-axis represents the SD (std. dev.; here and in subsequent figures) of the reach endpoint offsets from the target position, and thus lower values mean higher accuracy and better performance. The three boxes represent low, medium (Med.; here and in subsequent figures), and high dot scatter levels. Visual accuracy curves were estimated using VB condition data (Eq. 3), motor accuracy curves were estimated using MB condition data (Eq. 4), and CC endpoint accuracy was predicted by combining the visual and motor variability functions (Eq. 5). The solid, vertical line in each box indicates the reach initiation time that minimizes the $C\left(\right.$ condition performance, $\sigma_{c}$ (i.e., those $t_{\mathrm{v}}$ values that are predicted to yield maximum performance). Across dot scatter levels, the visual accuracy curves differ, and the $t_{\mathrm{v}}$ that is predicted to yield maximum performance increases (numbers with arrows).
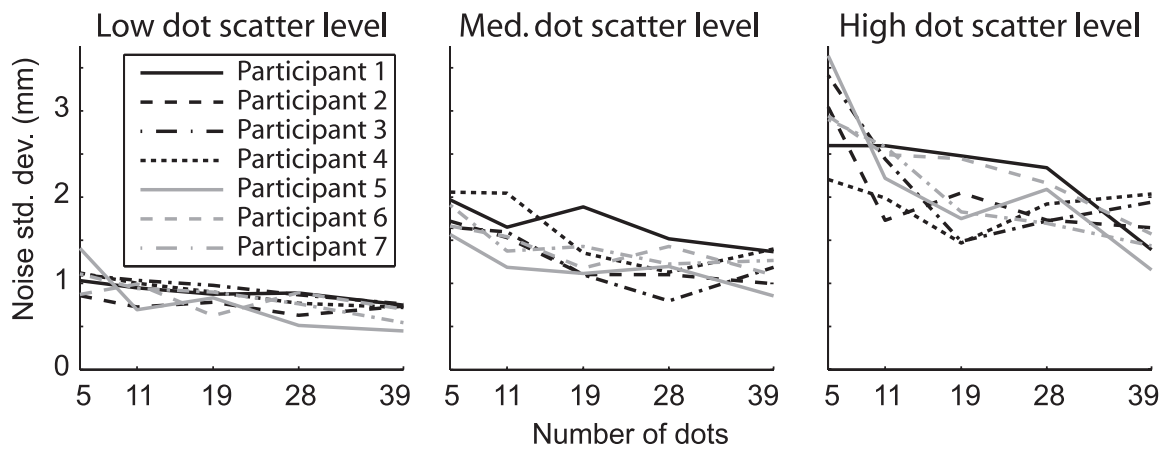

Figure 3. Dot centroid estimation noise (all participants). Each line represents the average error (quantified as a SD) of one participant's estimates of dot centroid locations in the VB condition. The $x$-axis represents different numbers of dots, and each box represents a different dot scatter level. Perfect estimation of dot centroids would correspond to noise SDs of 0 . In the Appendix, the dot centroid estimation noise was referred to as $\sigma_{\mathrm{e}}$.

$\mathrm{cm})$. The derivation of Equation 4 and the fitting procedure are described in the Appendix.

As mentioned above, in each CC condition trial, we allowed participants to choose a combination of $t_{\mathrm{V}}$ and $t_{\mathrm{M}}$ values. We will refer to the combination of $t_{\mathrm{V}}$ and $t_{\mathrm{M}}$ chosen on a single CC condition trial as $\left[t_{\mathrm{V}}\right.$, $\left.t_{\mathrm{M}}\right]$. Because the variance of the sum of two independent random variables is the sum of their variances, the variance of $\varepsilon_{\mathrm{C}}$, denoted by $\sigma_{\mathrm{C}}{ }^{2}$, is the sum of $\sigma_{\mathrm{V}}^{2}$ and $\sigma_{\mathrm{M}}{ }^{2}$ :

$$
\sigma_{\mathrm{C}}^{2}\left(t_{\mathrm{V}}, t_{\mathrm{M}}, \sigma_{\mathrm{d}}\right)=\sigma_{\mathrm{V}}^{2}+\sigma_{\mathrm{M}}^{2} .
$$

Note that the combined variance depends on both timing choices, $t_{\mathrm{V}}$ and $t_{\mathrm{M}}$, as well as the dot scatter level, $\sigma_{\mathrm{d}}$.

Because the VB and $\mathrm{MB}$ conditions measured how $t_{\mathrm{V}}, t_{\mathrm{M}}$, and $\sigma_{\mathrm{d}}$ affected participants' visual and motor variability independently, we used these relationships (Eq. 5) to formulate an ideal reacher model to predict how combining these sources of variability would affect participants' reach endpoint offsets in the CC condition. Specifically, by fitting the free parameters of ${\sigma_{\mathrm{V}}}^{2}$ and $\sigma_{\mathrm{M}}{ }^{2}$ (see Appendix), we were able to compute predicted $\sigma_{\mathrm{C}}$ landscapes that depended on $t_{\mathrm{V}}$ and $t_{\mathrm{M}}$, for each of the three experimental $\sigma_{\mathrm{d}}$ levels. Errors in fitted parameters (estimated by bootstrapping the data across MLE fits) were propagated by computing predictions for each set of bootstrap parameter fits. The ideal reacher is subject to the constraint that $t_{\mathrm{M}}$ is less than or equal to the remaining trial time after reach initiation $\left(1200-t_{\mathrm{V}}\right)$, just as in the CC condition. We assumed that participants learned the three $\sigma_{\mathrm{d}}$ levels from the practice and VB conditions and immediately implemented that knowledge in the CC condition.

For each $\sigma_{\mathrm{d}}$ level, we predicted ideal timing choices, $\left[t_{\mathrm{V}}{ }^{*}, t_{\mathrm{M}}{ }^{*}\right]$, by numerically minimizing Equation 5. Note that the predictions of our ideal model are based on the assumption that timing choices will be executed without error. However, we expect that humans viewing times and reaching times will differ from their choice intentions because of noise. Although we investigated the effects of noisy execution of timing choices on theoretical predictions, we found a full analysis provides only a modest increase in our ability to predict performance in the $\mathrm{CC}$ condition, and thus for simplicity, we ignored it.

\section{Results}

\section{Experiment 1}

We compared each human participant's performance in the CC task to their ideal predicted performance. A critical test is whether participant's choices vary with systematic changes in the quality of visual information (dot scatter level) as they do for the ideal predicted choices. To ensure our results reflected normal behavior and not learned associations, no performance feedback was given to participants in the reach task except a cumulative score presented every 30 trials (we later conducted Experiment 2 with performance feedback provided to assess what, if any, impact this had on performance).

\section{Baseline conditions}

The VB condition measured participants' $\sigma_{\mathrm{V}}$ functions. Because the number of dots, $N\left(t_{\mathrm{V}}\right)$, increased with viewing time, $t_{\mathrm{V}}$, and more dots gave more information about the target location, visual offsets should decrease as $t_{\mathrm{V}}$ increases. The dashed curves in Figure 2 show one participant's $\sigma_{\mathrm{V}}$ function, as described by the visual variability model (Eq. 3 ) with best-fit parameters. Figure 2 illustrates that as $t_{\mathrm{V}}$ increases, the participant's visual variability decreases.

Figure 3 depicts participants' errors in estimating dot centroid locations for various numbers of dots, $N\left(t_{\mathrm{V}}\right)$, and dot scatter levels, $\sigma_{\mathrm{d}}$, in the VB condition. These errors are computed relative to the mean, or centroid, of the dot scatter of each trial (rather than the underlying target location), to discount visual variability because of centroid misestimation. CC condition performance depends on the overall visual error, $\varepsilon_{\mathrm{V}}$, which combines the errors shown in Figure 3 with errors because of deviations between the centroid of the dots and the true target location. The relationship between the different components of participants' visual errors and $\sigma_{\mathrm{V}}$ is provided in the Appendix. Tassinari et al. (2006) reported a similar analysis of human dot centroid mislocaliza- 
Table 1. Motor precision model parameters (all participants)

\begin{tabular}{lc}
\hline & Mean \pm SEM \\
\hline$\alpha$ & $-1.213 \pm 0.36$ \\
$\beta$ & $0.271 \pm 0.062$ \\
$\gamma$ & $0.327 \pm 0.202$ \\
\hline
\end{tabular}

Parameter values of the motor precision model fit using MLE (Eq. 4) are shown. The SEM values represent SEMs computed by bootstrapped resampling of the raw data.

tions, and although they used slightly different dot scatter levels and numbers of dots, their results are consistent with ours when extrapolated to our particular conditions.

The MB condition measured each participant's motor precision (quantified as expected motor offset from the target location) as a function of $t_{\mathrm{M}}$. Remember that $t_{\mathrm{M}}$ is the remaining trial time after a reach initiation, so shorter viewing times, $t_{\mathrm{V}}$, allow greater movement times, $t_{\mathrm{M}}$. The dotted curves in Figure 2 show one participant's $\sigma_{\mathrm{M}}$ function, as described by our motor precision model (Eq. 4) with best-fit parameters. Figure 2 illustrates that as $t_{\mathrm{M}}$ increases (earlier reach initiations), participants' motor variability decreases. $\sigma_{\mathrm{M}}$ was used to predict the contribution of motor variability to $\sigma_{\mathrm{C}}$ in the CC condition. Table 1 represents mean $( \pm \mathrm{SEM})$ values for the fitted parameters from our motor precision model (Eq. 4).

\section{CC condition: timing choices}

Different dot scatter levels, $\sigma_{\mathrm{d}}$, produce different $\sigma_{\mathrm{C}}$ functions (Eq. 5). Because the minimal $\sigma_{\mathrm{C}}$ varies with dot scatter level, the ideal performer adjusts its $\left[t_{\mathrm{V}}{ }^{*}, t_{\mathrm{M}}{ }^{*}\right]$ choices for different values of $\sigma_{\mathrm{d}}$ (Fig. 2, solid, vertical lines with arrows and numbers indicating the $t_{\mathrm{V}}$ value). Specifically, the ideal performer should increase $t_{\mathrm{V}}$ and decrease $t_{\mathrm{M}}$ as dot scatter level increases. We compared each participant's actual timing choices to his or her respective ideal choice predictions for different dot scatter levels to assess how well humans chose to trade off visual and motor variability.

Figure 4 shows participant 4's measured $\left[t_{\mathrm{V}}, t_{\mathrm{M}}\right]$ choices from every CC condition trial (points) superimposed over the predicted $\sigma_{\mathrm{C}}$ landscape as a function of $\left[t_{\mathrm{V}}{ }^{*}, t_{\mathrm{M}}{ }^{*}\right]$ (grayscale contours). Lighter shades represent smaller values of $\sigma_{\mathrm{C}}$ from target location; darker shades represent larger values of $\sigma_{\mathrm{C}}$. We interpreted the mean of the observed timing choices as the participant's estimate of the timings that would maximize his or her performance (minimize reach endpoint offsets) and the covariance as resulting from variability in both decision-making and motor output.

To simplify the analysis and provide an intuitive measure of participants' timing choices, we computed the perpendicular projections of each $\left[t_{\mathrm{V}}, t_{\mathrm{M}}\right]$ vector onto the nearest point on the line with slope -1 and $y$-intercept $1200 \mathrm{~ms}$ (termed the total time axis) and called these values $t_{\mathrm{C}}$. The projections of the ideal performer's $\left[t_{\mathrm{V}}{ }^{*}, t_{\mathrm{M}}{ }^{*}\right]$ choices onto the total time axis are called $t_{\mathrm{C}}{ }^{*}$. The total time axis can be thought of as the set of timing choices for which $t_{\mathrm{V}}+t_{\mathrm{M}}=1200 \mathrm{~ms}$. Each $t_{\mathrm{C}}$ represents the nearest point on the total time axis to the participant's $\left[t_{\mathrm{V}}, t_{\mathrm{M}}\right]$ choice. This axis is meaningful, because the minimum of $\sigma_{\mathrm{C}}$ for ideal predictions shifted along that axis as a function of $\sigma_{\mathrm{d}}$.

Figure 5 shows a scatterplot of one participant's timing choices and their respective $t_{\mathrm{C}}$ values. The $x$-axis represents viewing time, the $y$-axis represents movement time, each dot represents a $\left[t_{\mathrm{V}}, t_{\mathrm{M}}\right]$ choice from one trial, and the diagonally aligned bar graph represents a histogram of $t_{\mathrm{C}}$ on the total time axis. Blue, green, and red dots/bars represent trials from the low, medium, and high dot scatter level conditions, respectively. Notice that as dot scatter level was increased, $t_{\mathrm{C}}$ choices shifted along the total time axis as $t_{\mathrm{V}}$ increased and $t_{\mathrm{M}}$ decreased.

We performed first-order linear regression analysis on the $t_{\mathrm{C}}$ values as a function of dot scatter level. Figure 6 summarizes the regression slopes [with 95\% confidence intervals (CIs)] for each participant. All slopes were significantly greater than zero $(p<$ $0.05)$, indicating all participants shifted $t_{\mathrm{C}}$ in the appropriate direction given $\sigma_{\mathrm{C}}$ across dot scatter levels.

Qualitatively, all participants adjusted their timing choices in concert with the ideal performer. A quantitative comparison between participants' actual $t_{\mathrm{C}}$ choices and their ideal performers' predicted $t_{\mathrm{C}}{ }^{*}$ choices is shown in Figure 7. For each participant, the means of measured $t_{\mathrm{C}}$ values were plotted against ideal performer's $t_{\mathrm{C}}{ }^{*}$ values that would minimize $\sigma_{\mathrm{C}}$.

Figure 7 confirms that all participants shifted their $t_{\mathrm{C}}$ values in the direction of the ideal predicted shift but sometimes with a lesser magnitude than predicted (see Discussion). One notable pattern in this figure is that all participants but one (participant 2) show a greater $t_{C}$ shift between high (Fig. 7 , rightmost points) and medium (middle points) dot scatter levels versus between medium and low (leftmost points) dot scatter levels. One potential explanation for this pattern is that participants may be less sensitive to their own internal visual target certainty at lower uncertainty levels. Another potential explanation may be an artifact of our methodology: we conducted the high dot scatter level condition several days after the low and medium dot scatter level conditions, the blocks of which were interleaved. Perhaps interleaving the low and medium scatter level blocks promoted strategy generalization among the participants in which they chose $\left[t_{\mathrm{V}}, t_{\mathrm{M}}\right] \mathrm{s}$ that were good for both scatter levels.

\section{CC condition: task performance}

To validate our predictive model of $\sigma_{\mathrm{C}}$ given baseline condition measurements, we compared participants' measured reach endpoints to their ideal performers' $\sigma_{\mathrm{C}}$ function. Figure 8 represents all participants' measured endpoint offset SDs plotted against the ideal predicted offset SDs. Each measured endpoint offset SD was computed by taking the SD of a participant's reach endpoint offsets for a particular dot scatter level, and the ideal predicted offset SD is simply the value of the ideal performer's predicted $\sigma_{\mathrm{C}}$ at its minimum. Figure 9 characterizes the relationship of predicted and measured performance by "combination efficiency." The percentages in Figure 9 were computed as follows: for each $\mathrm{CC}$ condition reach, the measured reach endpoint offset was divided by the value of $\sigma_{\mathrm{C}}$ at the participant's measured $\left[t_{\mathrm{V}}, t_{\mathrm{M}}\right]$ choice in that trial. We took the root mean square of these ratios and multiplied it by 100 to compute average efficiency, in percentage, of the participant's performance with respect to the model.

Generally, measured performances slightly exceeded predicted performance despite actual timing choices shifting slightly less across dot scatter levels than the ideal prediction. This slight overperformance may be attributable to increased participant motivation in the CC condition resulting from the increased difficulty of the task over the baseline conditions (Fig. 2) and greater allowance for participants' choices, because they chose $t_{\mathrm{V}}$ and $t_{\mathrm{M}}$ in the CC task as opposed to being cued to particular values of each, as in the MB and VB conditions.

\section{Experiment 2}

A final question addressed whether providing direct performance feedback to participants would substantially change their timing 

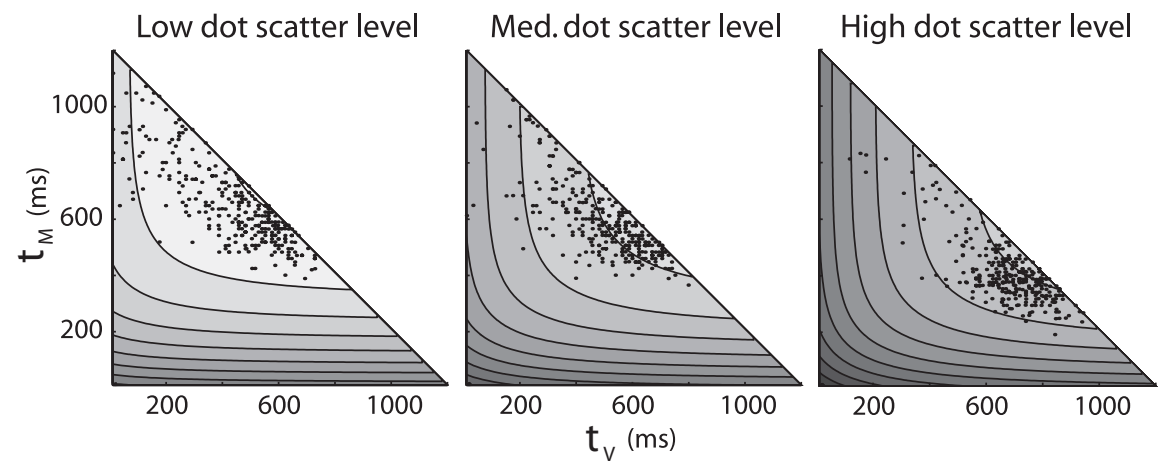

Figure 4. $\sigma_{\mathrm{C}}$ and measured $\left[t_{\mathrm{V}}, t_{\mathrm{M}}\right]$ choices (participant 4). Each of the three boxes depicts participant 4 's $\mathrm{CC}$ condition predicted $\sigma_{c}$ function for different dot scatter levels (grayscale contours), as well as measured $\left[t_{V}, t_{M}\right]$ choices (points). The $x$-axis represents $t_{\mathrm{V}}$ in milliseconds, and the $y$-axis represents $t_{M}$ in milliseconds. $\sigma_{c}$ is quantified as the $S D$ of reach endpoint offsets from the true target location (in millimeters). Dark contours represent large values of $\sigma_{C}$, and light contours represent small values of $\sigma_{c}$. Each point is a $\left[t_{v}, t_{M}\right]$ choice measured on a single trial.

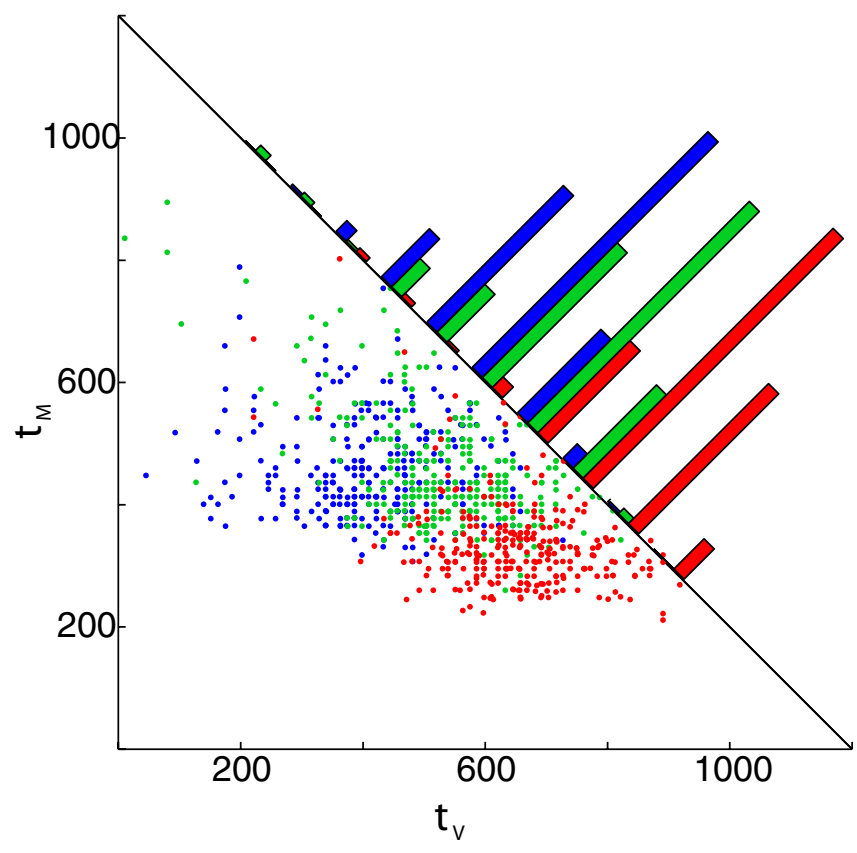

Figure 5. Timing choices and their corresponding $t_{c}$ values (participant 1). This figure is similar to Figure 4, but for participant 1. Instead of separating dot scatter levels into different boxes, we superimposed the $\left[t_{V}, t_{M}\right]$ choices from all dot scatter levels on each other and labeled them using different colors. The histogram represents the $t_{\mathrm{c}}$ values onto the total time axis (diagonal line). The $x$-axis represents $t_{v}$ in milliseconds, and the $y$-axis represents $t_{M}$ in milliseconds. The blue, green, and red points/bars are $\left[t_{V}, t_{M}\right]$ choices from the low, medium, and high dot scatter levels, respectively. The shifts between the histograms illustrate how the participant's timing choices varied across dot scatter levels.

choices, perhaps helping them choose $\left[t_{\mathrm{V}}, t_{\mathrm{M}}\right]$ nearer to the minimum of their ideal performer's $\sigma_{\mathrm{C}}$ function.

As described in Materials and Methods, Experiment 2 provided performance feedback indicating whether reaches were successful in the CC condition. If participants' timing choices remained unchanged between Experiments 1 and 2, we could conclude that participants did not require performance feedback to select $\left[t_{\mathrm{V}}, t_{\mathrm{M}}\right]$ with low values of $\sigma_{\mathrm{C}}$.

We found no significant difference in either CC task performance or $\left[t_{\mathrm{V}}, t_{\mathrm{M}}\right]$ distributions when direct feedback was provided. Figure 10 scatterplots CC task performance between Experiments 1 and 2. To quantify performance, we used the percentage of CC task trials in which the participant successfully reached the target. The $x$-axis represents Experiment 1 performances, and the $y$-axis represents Experiment 2 performances. Each point represents the performances for one dot scatter level, for one participant. The correlation between the points is $0.96(p<0.00001)$. This supports the view that each participant performed consistently across Experiments 1 and 2 .

Figure 11 scatterplots distributional areas of timing choices between Experiments 1 and 2 . To quantify distributional area, we used the square-root of the trace of the covariance matrix of each participant's $2 \mathrm{D}\left[t_{\mathrm{V}}, t_{\mathrm{M}}\right]$ distribution. We plotted the distributional areas for Experiment 1 against those for Experiment 2 to assess the consistency of participant's timing choice variability across the two experiments. The correlation between the points is 0.41 , which shows participants' $\left[t_{\mathrm{V}}, t_{\mathrm{M}}\right.$ ] distributions had similar areas, with and without direct feedback. If we consider the point with the "X" over it (participant 4, low dot scatter level condition) an outlier and remove it from this analysis, the correlation rises to 0.67 and becomes significantly different from zero $(p<0.05)$. Because there was little difference between distributional areas in Experiments 1 and 2, we conclude the variability in $\left[t_{\mathrm{V}}, t_{\mathrm{M}}\right]$ choices was unrelated to participants' knowledge of their own performance. Potential explanations for the timing variability are explored below.

\section{Discussion}

We interpreted our model and participants' behaviors in the context of statistical decision theory, whose application to sensorimotor neuroscience stems from a long tradition of treating perception and action as statistical computation problems (Attneave, 1954; Fitts, 1954; Barlow, 1961). Our results suggest that when performing visually guided motor behaviors, the brain represents both the quality of the visual information and potential motor output. Moreover, the brain understands how visual and motor variability depend on time and selects viewing and movement durations to minimize consequent errors.

These results are not necessarily surprising in isolation, as previous studies have shown human performance of various sensorimotor tasks reflects key elements of statistically optimal decision making (Maloney, 2002; Körding and Wolpert, 2006), including near-optimal use of sensory information (Kersten, 1987; Geisler, 1989, 2003; Legge et al., 1997; Knill, 1998; Kersten et al., 2004), reliability-weighted sensory information combination (Landy et al., 1995; Knill, 1998; Jacobs, 1999; Ernst and Banks, 2002; Battaglia et al., 2003; Alais and Burr, 2004; Ernst and Bülthoff, 2004; Shams et al., 2005), knowledge of the generative processes of sensory inputs (Knill and Kersten, 1991; Bloj et al., 1999; Battaglia et al., 2005), use of prior information (Mamassian and Landy, 2001; Weiss et al., 2002; Adams et al., 2004; Körding and Wolpert, 2004; Tassinari et al., 2006), internal motor output variability representations (Harris and Wolpert, 1998; Todorov and Jordan, 2002; Todorov, 2004), and selection of gainmaximizing actions (Schrater and Kersten, 2000; Trommershäuser et al., 2003a,b, 2005, 2006a,b; Wu et al., 2006). What is surprising is that these elements cooperate to allow human performance to approach optimal performance for a novel visuomotor task. Minimally, visual estimates and their respective accura- 


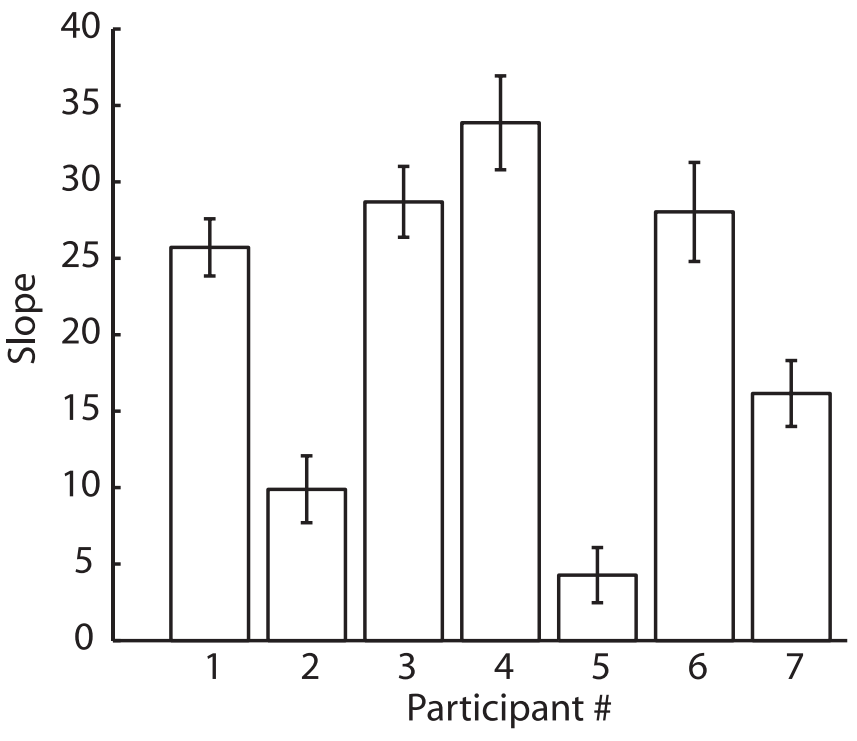

Figure 6. Regression slopes of $t_{c}$ values across dot scatter levels (all participants). Each bar represents the slope of the first-order linear regression of $t_{c}$ values on dot scatter level (with $95 \% \mathrm{Cls}$ ) for each participant. Every participant shifted his or her $\left[t_{\mathrm{V}}, t_{\mathrm{M}}\right]$ choices positively along the total time axis as dot scatter level was increased. This is qualitatively consistent with the shift predicted by the ideal performer model (Fig. 2, solid vertical lines).

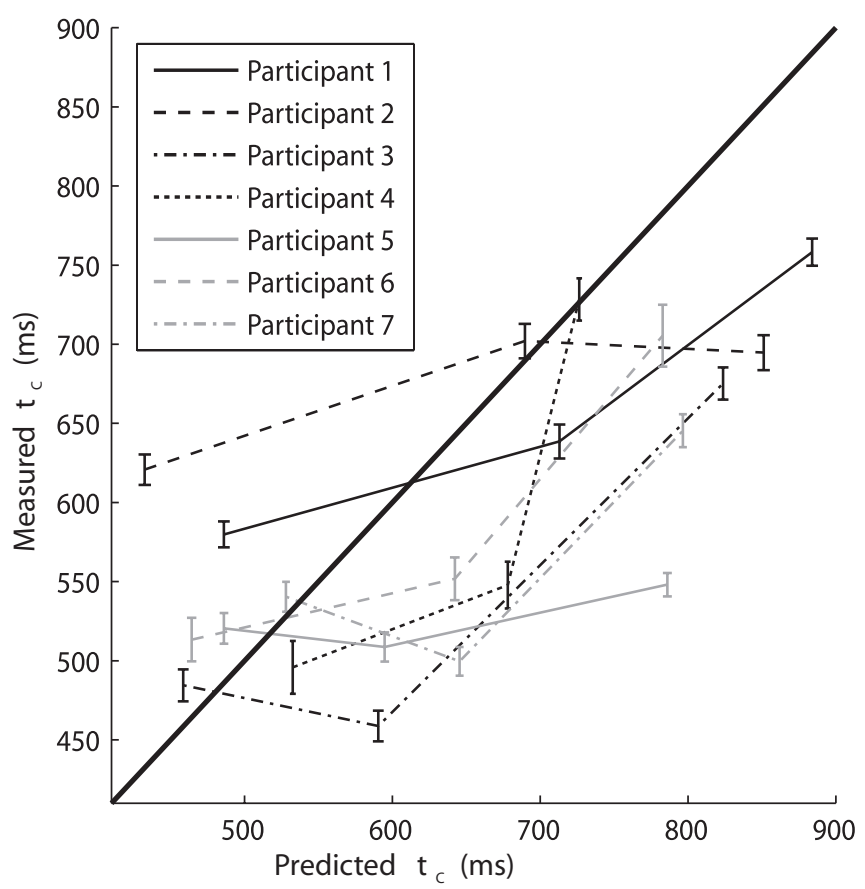

Figure 7. Measured versus predicted $t_{c}$ means (all participants). Measured $t_{c}$ means ( $y$ axis) are plotted against predicted $t_{c}{ }^{*}$ means $(x$-axis) for all participants. Each point represents one participant's $t_{c}$ mean (with $95 \% \mathrm{Cls}$ ) from a $\mathrm{CC}$ block with a particular dot scatter level. The various color and style combinations for the lines correspond to individual participants, as labeled by the legend. The heavy, solid, diagonal line represents perfect coherence between predicted and measured $t_{c}$ means.

cies are passed to motor-planning systems, which predict the precision of potential reaches and control viewing and movement timing to favorably trade off visual and motor variability.

Based on participants' isolated visual (VB) and motor (MB) performances, we predicted timing choices expected to minimize reaching errors in the combined visuomotor condition (CC). Participants clearly adjusted their timing choices in a manner

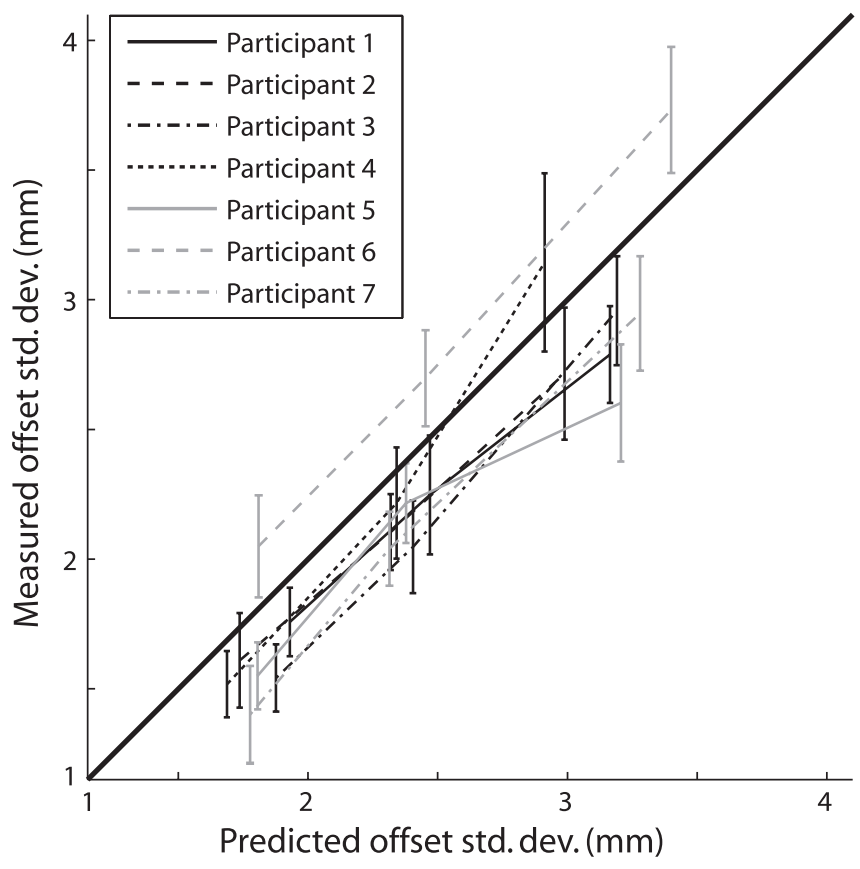

Figure 8. Measured versus predicted offset SDs (all participants). Measured reach endpoint offset SDs ( $y$-axis) are plotted against the predicted offset SDs at the predicted $\left[t_{V}, t_{\mathrm{M}}\right]$ choices ( $x$-axis). Each point represents the SDs of all offsets (with 95\% Cls) from a CC block with a particular dot scatter level. The various color and style combinations for the lines correspond to individual participants, as labeled by the legend. The heavy, solid, diagonal line represents perfect coherence between predicted and measured offset SDs.

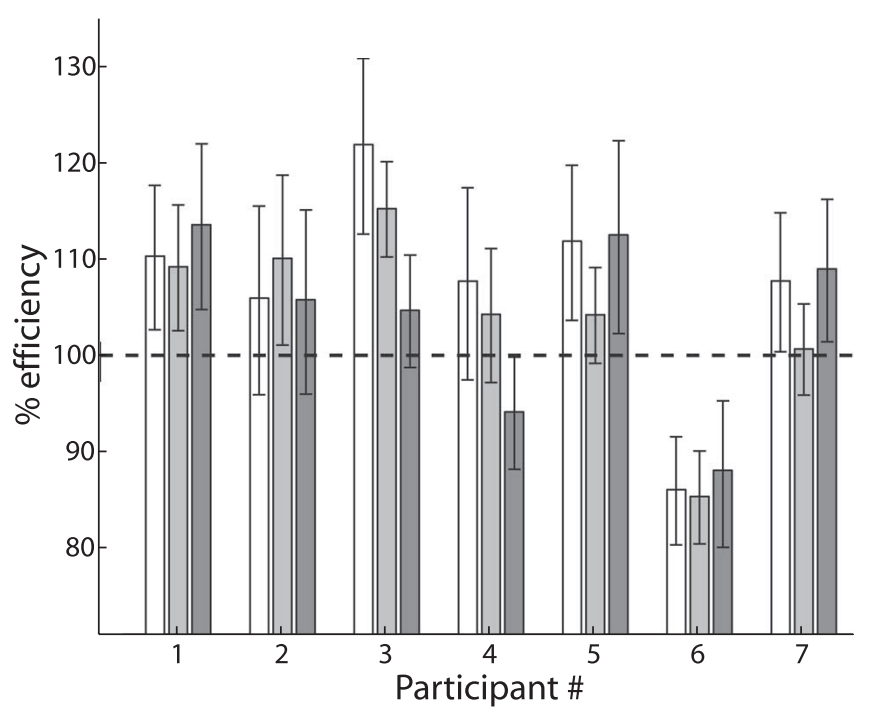

Figure 9. Measured reach endpoint accuracy with respect to predictions (all participants). Each triplet of bars is a set of efficiency scores for reach accuracy for a single participant (with $\pm 95 \%$ (ls). White, gray, and black bars are efficiency scores for the low, medium, and high dot scatter levels, respectively.

predicted to improve task performance. Because participants chose viewing and movement durations predicted to yield low offsets with nearly zero performance feedback, we conclude that this behavior is not merely an association between timings and success but in fact an internal representation of task structure. This conclusion is necessary to explain the different timing choice strategies across dot scatter levels. Because the additional direct performance feedback in Experiment 2 did not substantially improve (or otherwise modify) participants' behavior, it seems that 


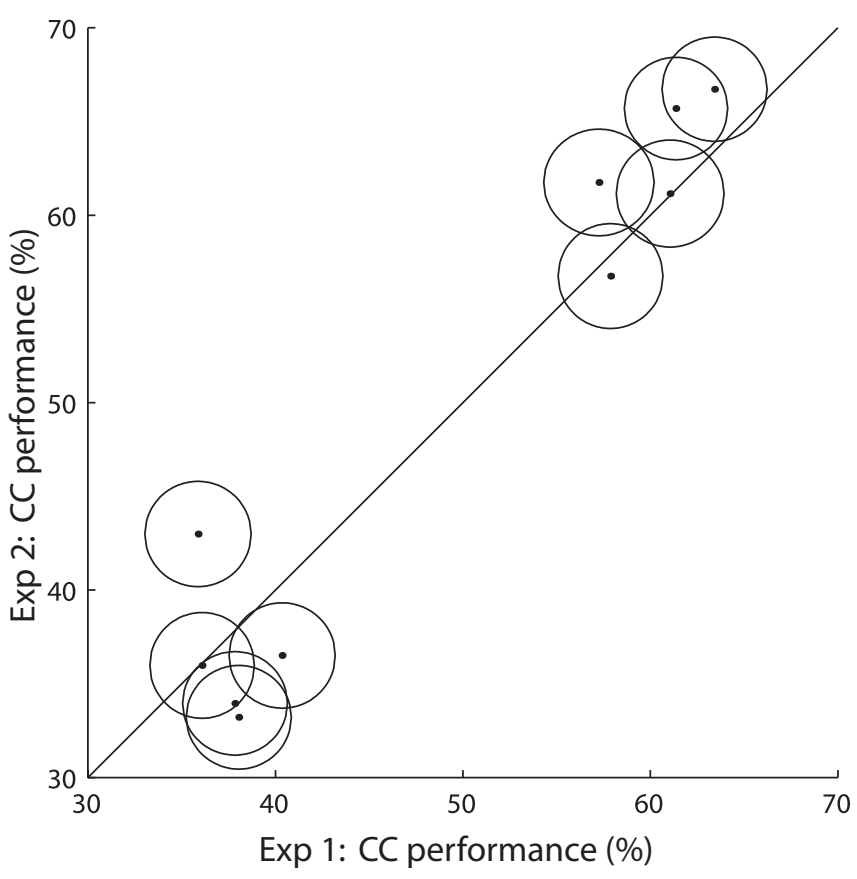

Figure 10. CC task performance comparison between Experiments 1 (Exp 1) and 2 (Exp 2; all Experiment 2 participants). Performances are expressed as percentage of total trials in which the participant successfully reached the target. The $x$-axis represents Experiment 1 performances, and the $y$-axis represents Experiment 2 performances. Each point represents Experiments 1 and 2 performances for one dot scatter level for one participant. The ellipses are SEM ellipses in both $x$ and $y$ directions, as computed by bootstrapped resampling of the raw data. The correlation between the points is $0.96(p<0.00001)$.

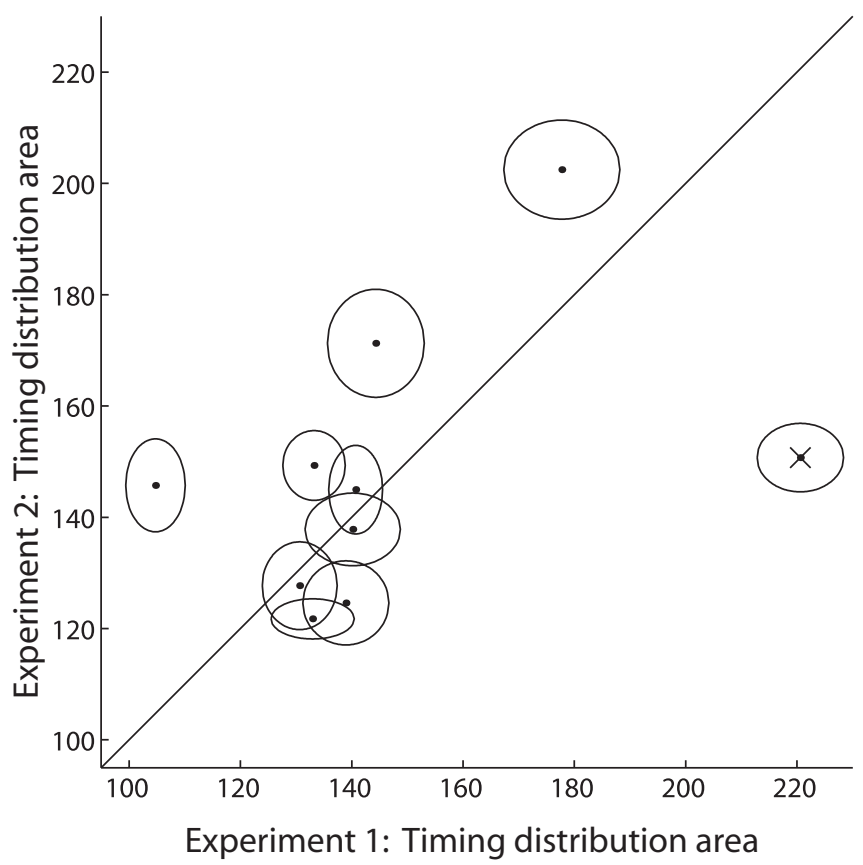

Figure 11. Timing distributional area comparison between Experiments 1 and 2 (all Experiment 2 participants). Timing distributional area was quantified as the square-root of the trace of the covariance matrix of the $\left[t_{V}, t_{M}\right]$ choice distribution of each dot scatter level. The $x$-axis represents Experiment $1\left[t_{v}, t_{M}\right]$ distributional area, and the $y$-axis represents Experiment 2 distributional area. Each point represents the distributional areas of the two experiments for one dot scatter level for one participant. The ellipses are SEM ellipses in both $x$ and $y$ directions, as computed by bootstrapped resampling of the raw data. The point with the $X$ over it (participant 4; low dot scatter level condition) can be considered an outlier. inherent knowledge of visual and motor variability are sufficient to support optimal task performance, and little learning is required to optimize that knowledge. A potential future experiment could impose an alternative manipulation of the optimal trade-off time by varying the rate at which new dots appear. If carefully controlled, this manipulation could help expose the relationship between neural integration windows and information accumulation periods for perceptual decision making (Mazurek et al., 2003; Uchida et al., 2006).

One puzzling feature of our data is the variability in participants' measured timing choices in the CC condition. Across participants, the SDs of $t_{\mathrm{M}}$ measured in the $\mathrm{MB}$ condition were consistent with those of Zelaznik et al. (1988). The average SD of $t_{\mathrm{M}}$ measured in the CC condition across participants was $98.9 \mathrm{~ms}$ ( $\pm 18.0 \mathrm{~ms} ; 95 \% \mathrm{CI}$ ), which was 1.8 times (with 1.3 as the $95 \%$ lower confidence interval) our MB measurements, as well as those of Zelaznik et al. (1988), predict as participants' minima. This means participants allowed significantly more $(p<0.05)$ variability in movement time, $t_{\mathrm{M}}$, in the CC condition than in the $\mathrm{MB}$ condition. We conclude that participants did not deem $t_{\mathrm{M}}$ variance minimization to be critically important in the CC condition. Likewise, CC condition viewing times, $t_{\mathrm{V}}$, had high variability, with an average SD of $122.1 \mathrm{~ms}( \pm 22.7 \mathrm{~ms} ; 95 \% \mathrm{CI})$ across participants.

We could not concretely explain these high degrees of variability, but there are several possibilities. First, participants' choices may reflect a principle from learning theory called the exploration/exploitation tradeoff. This holds that when learning to improve task performance, optimal task behavior may be deliberately sacrificed to test whether novel behaviors may potentially yield greater performance. In our task, participants may choose novel $\left[t_{\mathrm{V}}, t_{\mathrm{M}}\right]$ values that are not consistent with their estimates of the optimal $\left[t_{\mathrm{V}}{ }^{*}, t_{\mathrm{M}}{ }^{*}\right]$ choices to investigate whether these novel timings may improve performance. Despite the lack of performance feedback in Experiment 1 beyond the 30 trial cumulative scores, participants may internally monitor their reach performance to provide supervision for learning, akin to "bootstrapped-learning." A second possibility is that participants have some uncertainty about the exact $t_{\mathrm{V}}$ and $t_{\mathrm{M}}$ combinations that minimize $\sigma_{\mathrm{C}}$. When people are uncertain which timing is best, they may simply consider a set of timings to be "good enough" and thus explicitly allow $\left[t_{\mathrm{V}}, t_{\mathrm{M}}\right]$ choices to vary within that set. This is qualitatively consistent with the "minimum intervention principle" posited by Todorov and Jordan (2003). A third possibility is that because $\sigma_{\mathrm{C}}$ have relatively flat minima, the predicted difference in monetary reward is so small, perhaps as small as a few cents, that the cost of controlling $\left[t_{\mathrm{V}}, t_{\mathrm{M}}\right]$ choices is higher than the small payoff such control may yield.

A potentially related issue was the smaller-than-predicted timing shifts across dot scatter levels. One possibility again relates to participants devoting some trials to task exploration, as described above. In this case, the extreme dot scatter levels (e.g., low and high) would have some $\left[t_{\mathrm{V}}, t_{\mathrm{M}}\right]$ choices distributed toward the middle of the available timing range, thus lowering the measured shifts across scatter levels. A second possibility involves a potential mismatch between the assumptions in the ideal reacher model and human behavior. Ideal timing choices contain no temporal scatter and have no restrictions on $t_{\mathrm{M}}$ (e.g., $t_{\mathrm{M}}=0$ is possible). Perhaps in the presence of temporal scatter and additional unmodeled costs associated with extremely rapid movements, the range of admissible $t_{\mathrm{M}}$ choices was reduced, consequently reducing the range of $t_{\mathrm{V}}$ choices as well. A third possibility is that, again, because of the relatively flat minimum of 
$\sigma_{\mathrm{C}}$, participants may not expect sufficient reward to warrant fully changing their choice strategy with dot scatter level.

One limitation of our model is that it only places cost on missing the target. The model does not acknowledge any cost for timing out. Therefore, the optimal $\left[t_{\mathrm{V}}{ }^{*}, t_{\mathrm{M}}{ }^{*}\right]$ choices of our model always lie on the total trial time axis (slope, -1 ; $y$-intercept, $1200 \mathrm{~ms}$ ). Timing choices that lie on the total trial time axis imply that the participant used all $1200 \mathrm{~ms}$ of the total trial time (i.e., $t_{\mathrm{V}}+t_{\mathrm{M}}=1200 \mathrm{~ms}$ ). This means that if the participant's $t_{\mathrm{V}}$ or $t_{\mathrm{M}}$ lasted even $1 \mathrm{~ms}$ longer, the trial would time out. Because people cannot control the duration of $t_{\mathrm{V}}$ or $t_{\mathrm{M}}$ to within $1 \mathrm{~ms}$, the optimal decision is to shorten $t_{\mathrm{V}}$ and/or $t_{\mathrm{M}}$ to avoid timeout costs. In our experiment, participants often shortened $t_{\mathrm{V}}$ and/or $t_{\mathrm{M}}$ such that $t_{\mathrm{V}}+t_{\mathrm{M}}<1200 \mathrm{~ms}$. We believe implicit timeout costs explain why many timing choices are displaced away from the total time axis. Although we did not impose a monetary penalty for timeouts, and each timeout trial is repeated later, it is reasonable to assume participants would prefer to avoid repeating timeout trials. We applied simple estimates of timeout costs to our ideal performer model, but the estimates were inaccurate and participants' consistency with these timeoutpenalized optimal timing choices only improved marginally, so we did not report those analyses.

Although our experiment was purely psychophysical, neurophysiological studies support the existence of neural representations and computations required for optimal task performance, including time, probability, and reward-driven decision making (Schall, 2001; Platt, 2002; Sugrue et al., 2005). Recent studies have provided evidence for temporal probability representations in lateral intraparietal areas (LIPs) and parietal cortex in general. Leon and Shadlen (2003) reported evidence of neurons in macaque LIP that code the value as well as uncertainty of a remembered temporal duration (or at least monkeys' judgments of such quantities), whereas Janssen and Shadlen (2005) reported evidence of macaque LIP neurons encoding the probability of the occurrence of an event as a function of time. These results highlight potential cortical substrates for processing components our task requires, specifically representing trial time to decide when, and for how long, to execute reach movements.

In our task, the brain represents visual and motor uncertainty relationships and combines them to perform a joint task. A natural question is whether such behavior relies on cortically separate visual and motor representations or a unified representation of visuomotor uncertainty. Compelling arguments exist for both of these views. Coordinate transformations, temporal syncing, and the propagation of task goals to individual visual and motor decisions may be better served by a unified representation of visuomotor uncertainty. Conversely, independent noise corruption, sensory or motor recalibration, and general organizational simplicity may favor separate representations (for review, see Pouget et al., 2002).

In conclusion, our results supported the view that people can represent their visual and motor variability as functions of time. Moreover, they can combine these components to predict their performance in a task that depends on both, and select viewing and movement durations to minimize reaching errors. This behavior is consistent with Bayesian Decision Theoretic performance of visuomotor tasks.

\section{Appendix}

\section{Visual variability model}

We assumed that participants computed $\hat{X}$ by estimating the centroid of the dot positions by taking their mean. The overall devi- ation of $\hat{X}$ from $X$ was given by $\varepsilon_{\mathrm{V}}$, as mentioned, but this term can be further split into two discrete sources of error such that

$$
\varepsilon_{\mathrm{v}}=\varepsilon_{\mu}+\varepsilon_{\mathrm{e}}
$$

$\varepsilon_{\mathrm{V}}$ has mean equal to 0 , and variance, $\sigma_{\mathrm{V}}{ }^{2}$, which is the sum of the variances of $\varepsilon_{\mu}$ and $\varepsilon_{\mathrm{e}}$ (by conditional independence) such that

$$
\sigma_{\mathrm{v}}^{2}=\sigma_{\mu}^{2}+\sigma_{e}^{2}
$$

The first source of error, $\varepsilon_{\mu}$, results from deviations between the mean of the dot positions, $\mu_{\mathrm{d}}\left(t_{\mathrm{V}}\right)$, and $X$, resulting from randomness in sampling the dot positions. Because the dot positions were normally distributed, $\varepsilon_{\mu}$ was normally distributed with mean 0 and SD $\sigma_{\mu}$. Formally, $\sigma_{\mu}$ was a function of viewing time, $t_{\mathrm{V}}$, and dot scatter level $\sigma_{\mathrm{d}}$ such that

$$
\sigma_{\mu}\left(\mathrm{t}_{\mathrm{v}}, \sigma_{\mathrm{d}}\right)=\frac{\sigma_{\mathrm{d}}}{\sqrt{N\left(\mathrm{t}_{\mathrm{v}}\right)}},
$$

using $N\left(t_{\mathrm{V}}\right)$ from Equation 1. Notice that there are no free parameters to fit from data.

The second source of uncertainty, $\varepsilon_{\mathrm{e}}$, results from participants' misestimates of the positions of individual dots, which we assumed were corrupted by independent, mean 0 , normally distributed positional uncertainty. Therefore, $\varepsilon_{\mathrm{e}}$ was normally distributed with mean 0 and SD $\sigma_{\mathrm{e}}$. For simplicity, $\sigma_{\mathrm{e}}$ was assumed to be a first-order linear function of $\sigma_{\mu}$ and thus dependent on $t_{\mathrm{V}}$ and $\sigma_{\mathrm{d}}$ as well such that

$$
\sigma_{\mathrm{e}}\left(\mathrm{t}_{\mathrm{v}}, \sigma_{\mathrm{d}}\right)=\rho_{\mathrm{e}} \cdot \sigma_{\mu}+\omega_{\mathrm{e}},
$$

where $\rho_{\mathrm{e}}$ and $\omega_{\mathrm{e}}$ were free parameters.

By substituting Equations $\mathrm{A} 3$ and $\mathrm{A} 4$ into $\mathrm{A} 2, \varepsilon_{\mathrm{V}}$ has mean equal to 0 , and variance $\sigma_{\mathrm{V}}^{2}$ such that

$$
\sigma_{\mathrm{v}}\left(\mathrm{t}_{\mathrm{v}}, \sigma_{\mathrm{d}}\right)^{2}=\left(\rho_{e}^{2}+1\right) \frac{\sigma_{d}^{2}}{\mathrm{~N}\left(\mathrm{t}_{\mathrm{v}}\right)}+2 \rho_{e} \omega_{e} \frac{\sigma_{d}}{\sqrt{N\left(\mathrm{t}_{\mathrm{v}}\right)}}+\omega_{e}^{2},
$$

as given in Equation 3 in Materials and Methods. We fit the free parameters, $\rho_{\mathrm{e}}$ and $\omega_{\mathrm{e}}$, by MLE using the VB data separately for each participant, for each dot scatter level, as described below.

\section{Motor variability model}

We assumed that errors between $\hat{X}$ and $Z, \varepsilon_{\mathrm{M}}$, were attributable to motor noise and normally distributed, with mean 0 , and $\mathrm{SD} \sigma_{\mathrm{M}}$. Classically, Fitt's law expresses a relationship between average movement duration and target width that has several variants (MacKenzie and Buxton, 1992). It has been used to model reach endpoint SDs as a function of movement durations (Schmidt et al., 1978; Harris and Wolpert, 1998). We modified the simplest form of the equation as follows:

$$
T=a+b \cdot \log \left(\frac{D}{W}\right)
$$

where $T$ is expected movement duration, $D$ is target distance, and $W$ is target width. $a$ and $b$ are free parameters that vary across tasks and participants.

In particular, we characterized $\sigma_{\mathrm{M}}$ as a function of $t_{\mathrm{M}}$, or $\sigma_{\mathrm{M}}\left(t_{\mathrm{M}}\right)$. We related $W$ to $\varepsilon_{\mathrm{M}}$ by observing that $95 \%$ of reach endpoints will lie within the target width when

$$
\sigma_{\mathrm{M}}\left(t_{\mathrm{M}}\right)=\frac{W}{1.96}
$$


Note that we have equated the expected movement time, $T$, with the movement time choice, $t_{\mathrm{M}}$. To formulate endpoint variability as a function of $t_{\mathrm{M}}$, we solve the above expression for $W$, replace $W$ with $\sigma_{\mathrm{M}} / c$, where $c$ appropriately scales the endpoint variability to match success criterion for acquiring the target (i.e., the target width). Also, we add a constant, $\gamma$, that represents the minimum achievable movement endpoint offset $(\gamma$ can be thought of as the offset achievable if given a very long time to complete the reach). The resulting expression is fit to individual participant data:

$$
\sigma_{\mathrm{M}}\left(t_{\mathrm{M}}\right)=\mathrm{D} \cdot \exp \left(\alpha-\beta t_{\mathrm{M}}\right)+\gamma
$$

as given by Equation 4 in Materials and Methods, where

$$
\beta=\frac{1}{b}, \alpha=\frac{a}{b}+\log (c)
$$

absorbs the effects of $c$. We fit the free parameters $\alpha, \beta$, and $\gamma$ using MLE as described below.

\section{Maximum likelihood estimation of model parameters}

MLE of model parameters was separately fit for each participant using the baseline condition data. The log-likelihood of the data was computed for each reach by evaluating the probability of the offset of each trial using the model, evaluated at various model parameter values. Those model parameters that produced the maximum likelihood were considered to be best-fit parameters. For example, the likelihood of $\{\alpha, \beta, \gamma\}$ is given by the following expression, where $\phi(\cdot)$ is a Gaussian density function, $Y_{\mathrm{i}}$ is the offset for the $i^{\text {th }}$ reach, and $k$ is the total number of reaches:

$$
L(\alpha, \beta, \gamma)=\sum_{i=1}{ }^{k} \log \left[\phi\left(Y_{i} ; 0,\left(D \cdot \exp \left(\alpha-\beta \cdot t_{\mathrm{M}}\right)+\gamma\right)^{2}\right)\right]
$$

The likelihood for the visual data is similar. These expressions were numerically maximized to find optimal parameters.

\section{Bootstrapped confidence intervals/SEMs}

Reported results are accompanied by $95 \%$ confidence intervals or SEMs. When necessary for predictions, these confidence intervals or SEMs were computed by bootstrapped resampling of the raw data (Efron and Tibshirani, 1994). Specifically, we sampled the original data with replacement 50 times (or more if computational costs were not prohibitive) and performed the reported analysis on all 50 resampled data sets. From the set of 50 results, we computed the mean and SD; the mean is the value reported, and the bootstrap SD represents the SEM 95\% confidence intervals were computed as 1.96 times the SEM.

\section{References}

Adams WJ, Graf EW, Ernst MO (2004) Experience can change the 'lightfrom-above' prior. Nat Neurosci 7:1057-1058.

Alais D, Burr D (2004) The ventriloquist effect results from near-optimal bimodal integration. Curr Biol 14:257-262.

Attneave F (1954) Some informational aspects of visual perception. Psychol Rev 61:183-193.

Barlow HB (1961) The coding of sensory messages. In: Current problems in animal behaviour (Thorpe WH, Zangwill OL, eds), pp 331-360. Cambridge: Cambridge UP.

Battaglia PW, Jacobs RA, Aslin RN (2003) Bayesian integration of visual and auditory signals for spatial localization. J Opt Soc Am A 20:1391-1397.

Battaglia PW, Schrater PR, Kersten DJ (2005) Auxiliary object knowledge influences visually-guided interception behavior. Proceedings of the 2nd symposium on applied perception in graphics and visualization, ACM
International Conference Proceeding Series, A Coruña, Spain, August. 95:145-152.

Bloj M, Kersten DJ, Hurlbert AC (1999) 3D shape perception influences colour perception via mutual illumination. Nature 402:877-879.

Efron B, Tibshirani RJ (1994) An introduction to the bootstrap. New York: Chapman and Hall.

Ernst MO, Banks MS (2002) Humans integrate visual and haptic information in a statistically optimal fashion. Nature 415:429-433.

Ernst MO, Bülthoff HH (2004) Merging the senses into a robust percept. Trends Cogn Sci 8:162-169.

Fitts PM (1954) The information capacity of the human motor system in controlling the amplitude of movement. J Exp Psychol 47:381-391.

Geisler WS (1989) Sequential ideal-observer analysis of visual discriminations. Psychol Rev 96:267-314.

Geisler WS (2003) Ideal observer analysis. In: The visual neurosciences (Chalupa L, Werner J, eds), pp 825-837. Boston: MIT.

Harris CM, Wolpert DM (1998) Signal-dependent noise determines motor planning. Nature 394:725-726.

Jacobs RA (1999) Optimal integration of texture and motion cues to depth. Vision Res 39:3621-3629.

Janssen P, Shadlen MN (2005) A representation of the hazard rate of elapsed time in macaque area LIP. Nat Neurosci 8:234-241.

Kersten D (1987) Statistical efficiency for the detection of visual noise. Vis Res 27:1029-1040.

Kersten D, Mamassian P, Yuille A (2004) Object perception as Bayesian inference. Annu Rev Psychol 55:271-304.

Knill DC (1998) Ideal observer perturbation analysis reveals human strategies for inferring surface orientation from texture. Vision Res 38:2635-2656.

Knill DC, Kersten D (1991) Apparent surface curvature affects lightness perception. Nature 351:228-229.

Körding KP, Wolpert DM (2004) Bayesian integration in sensorimotor learning. Nature 427:244-247.

Körding KP, Wolpert DM (2006) Bayesian decision theory in sensorimotor control. Trends Cogn Sci 10:320-326.

Landy MS, Maloney LT, Johnsten EB, Young M (1995) Measurement and modeling of depth cue combinations: in defense of weak fusion. Vision Res 35:389-412.

Legge GE, Klitz TS, Tjan BS (1997) Mr. Chips: an ideal-observer model of reading. Psychol Rev 104:524-553.

Leon MI, Shadlen MN (2003) Representation of time by neurons in the posterior parietal cortex of the macaque. Neuron 38:317-327.

MacKenzie IS, Buxton W (1992) Extending Fitts' law to two-dimensional tasks. In: Proceedings of the CHI '92 conference on human factors in computing systems, pp 219-226. New York: ACM.

Maloney L (2002) Statistical decision theory and biological vision. In: Perception and the physical world: psychological and philosophical issues in perception (Heyer D, Mausfeld R, eds), pp 145-189. New York: Wiley.

Mamassian P, Landy MS (2001) Interaction of visual prior constraints. Vision Res 41:2653-2688.

Mazurek ME, Roitman JD, Ditterich J, Shadlen MN (2003) A role for neural integrators in perceptual decision making. Cereb Cortex 13:1257-1269.

Platt ML (2002) Neural correlates of decisions. Curr Opin Neurobiol 12:141-148.

Pouget A, Deneve S, Duhamel JR (2002) A computational perspective on the neural basis of multisensory spatial representations. Nat Rev Neurosci 3:741-747.

Schall JD (2001) Neural basis of deciding, choosing, and acting. Nat Rev Neurosci 2:33-42.

Schmidt RA, Zelaznik HN, Frank JS (1978) Sources of inaccuracy in rapid movement. In: Information processing in motor control and learning (Stelmach GE, ed), pp 183-203. New York: Academic.

Schrater PR, Kersten D (2000) How optimal depth cue integration depends on the task. Int J Comp Vis 40:71-89.

Shams L, Ma WJ, Beierholm U (2005) Sound-induced flash illusion as an optimal percept. NeuroReport 16:1923-1927.

Sugrue LP, Corrado GS, Newsome WT (2005) Choosing the greater of two goods: neural currencies for valuation and decision making. Nat Rev Neurosci 16:1-13.

Tassinari H, Hudson TE, Landy MS (2006) Combining priors and noisy visual cues in a rapid pointing task. J Neurosci 26:10154-10163. 
Todorov E (2004) Optimality principles in sensorimotor control. Nat Neurosci 7:907-915.

Todorov E, Jordan M (2002) Optimal feedback control as a theory of motor coordination. Nat Neurosci 5:1226-1235.

Todorov E, Jordan M (2003) A minimal intervention principle for coordinated movement. In: Advances in neural information processing systems 15 (Becker S, Thrun S, Obermayer K, eds), pp 27-34. Boston: MIT.

Trommershäuser J, Maloney LT, Landy MS (2003a) Statistical decision theory and trade-offs in the control of motor response. Spat Vis 16:255-275.

Trommershäuser J, Maloney LT, Landy MS (2003b) Statistical decision theory and the selection of rapid, goal-directed movements. J Opt Soc Am A 20:1419-1433.

Trommershäuser J, Gepshtein S, Maloney LT, Landy MS, Banks MS (2005) Optimal compensation for changes in task-relevant movement variability. J Neurosci 25:7169-7178.
Trommershäuser J, Landy MS, Maloney LT (2006a) Humans rapidly estimate expected gain in movement planning. Psychol Sci 17:981-988.

Trommershäuser J, Mattis J, Maloney LT, Landy MS (2006b) Limits to human movement planning with delayed and unpredictable onset of needed information. Exp Brain Res 175:276-284.

Uchida N, Kepecs A, Mainen ZF (2006) Seeing at a glance, smelling in a whiff: rapid forms of perceptual decision making. Nat Rev Neurosci 7:485-491.

Weiss Y, Simoncelli EP, Adelson EH (2002) Motion illusions as optimal percepts. Nat Neurosci 5:598-604.

Wu SW, Trommershäuser J, Maloney LT, Landy MS (2006) Limits to human movement planning in tasks with asymmetric value landscapes. J Vision 6:53-63.

Zelaznik HN, Mone S, McCabe GP, Thaman C (1988) Role of temporal and spatial precision in determining the nature of the speed-accuracy trade-off in aimed hand movements. J Exp Psychol Hum Percept Perform 14:221-230. 